This document is published in:

Kurachi, C., Svanberg, K. \& Tromberg, B. J. (eds.) (2015). Biophotonics South America. Proceedings SPIE, vol . 9531, 9531111. Society of PhotoOptical Instrumentation Engineers

DOI: http://dx.doi.org/10.1117/12.2189960

(C) 2015. Society of Photo-Optical Instrumentation Engineers. One print or electronic copy may be made for personal use only. Systematic electronic or print reproduction and distribution, duplication of any material in this paper for a fee or for commercial purposes, or modification of the content of the paper are prohibited. 


\title{
Fluorescence multi-scale endoscopy and its applications in the study and diagnosis of gastro-intestinal diseases: set-up design and software implementation
}

\author{
Pablo Aurelio Gómez-García ${ }^{1,2}$, Alicia Arranz ${ }^{3}$, Manuel Fresno ${ }^{3}$, Manuel Desco ${ }^{12}$, Umar \\ Mahmood $^{4}$, Juan José Vaquero ${ }^{1,2}$, Jorge Ripoll ${ }^{1,2}$ \\ ${ }^{1}$ Department of Bioengineering and Aerospace Engineering, Universidad Carlos III de Madrid, \\ 28911 Madrid, Spain \\ ${ }^{2}$ Experimental Medicine and Surgery Unit, Instituto de Investigación Sanitaria del Hospital \\ Gregorio Marañón, 28007 Madrid, Spain. \\ ${ }^{3}$ Department of Cell Biology and Immunology, Center for Molecular Biology "Severo Ochoa" \\ (CBMSO), CSIC, Madrid, Spain. \\ ${ }^{4}$ Athinoula A. Martinos Center, Massachusetts General Hospital, Charlestown, MA 02129, U.S.A.
}

\begin{abstract}
Endoscopy is frequently used in the diagnosis of several gastro-intestinal pathologies as Crohn disease, ulcerative colitis or colorectal cancer. It has great potential as a non-invasive screening technique capable of detecting suspicious alterations in the intestinal mucosa, such as inflammatory processes. However, these early lesions usually cannot be detected with conventional endoscopes, due to lack of cellular detail and the absence of specific markers. Due to this lack of specificity, the development of new endoscopy technologies, which are able to show microscopic changes in the mucosa structure, are necessary. We here present a confocal endomicroscope, which in combination with a wide field fluorescence endoscope offers fast and specific macroscopic information through the use of activatable probes and a detailed analysis at cellular level of the possible altered tissue areas. This multi-modal and multi-scale imaging module, compatible with commercial endoscopes, combines near-infrared fluorescence (NIRF) measurements (enabling specific imaging of markers of disease and prognosis) and confocal endomicroscopy making use of a fiber bundle, providing a cellular level resolution. The system will be used in animal models exhibiting gastro-intestinal diseases in order to analyze the use of potential diagnostic markers in colorectal cancer. In this work, we present in detail the set-up design and the software implementation in order to obtain simultaneous RGB/NIRF measurements and short confocal scanning times.
\end{abstract}

Keywords: Endoscopy, Endomicroscopy, Fiber bundle, Fluorescence, Scan, Software, Cell imaging.

\section{INTRODUCTION}

Endoscopy is used in the diagnosis of gastrointestinal conditions such as inflammatory bowel disease, Crohn's disease, ulcerative colitis or colorectal cancer, and there are a great number of different endoscopy systems with its corresponding applications $[1,2,3]$. Diagnostic endoscopy is also usually accompanied by the collection of biopsies which are then analyzed by standard histological techniques. Endoscopy also has great potential as a technique for early detection of alterations in the intestinal mucosa, which can then lead to chronic inflammatory processes or neoplasms. Early detection allows early onset of specific treatment and the possibility of reducing the severity of the possible complications of the disease. However, such early lesions are not usually detected with conventional endoscopes. The main reason is the lack of cellular detail offered by these endoscopes and lack of markers with specific contrast. The latest advances in endoscopy, mainly video-endoscopy, high resolution endoscopy, and narrow band imaging, attempt to improve this situation, but are still far from providing the specificity and resolution required, and are not suitable for the study of molecular markers. Therefore, it is essential to develop more advanced endoscopic technologies that enable observation of gastrointestinal epithelium with higher resolution, making obvious microscopic changes in the structure of the mucosa, which would otherwise go undetected with conventional endoscopic techniques. Such is the case of confocal endomicroscopy, which potentially offers the possibility of a histological analysis in vivo and in situ, thereby enabling rapid diagnosis also can result in immediate intervention by the physician.

Biophotonics South America, edited by Cristina Kurachi, Katarina Svanberg, Bruce J. Tromberg,

Vanderlie Salvador Bagnato, Proc. of SPIE Vol 9531, 953111 • C) 2015 SPIE

CCC code: $1605-7422 / 15 / \$ 18 \cdot$ doi: $10.1117 / 12.2189960$

Proc. of SPIE Vol. $9531953111-1$ 
Nowadays video-endoscopy is the most used technique to detect and aid in the diagnosis of gastrointestinal diseases. This detection is based primarily on interpreting images in color (RGB) provided by the video. The diagnosis thus is based on pigmentation changes in the geometry or the displayed area. Due to the low specificity of these techniques, three main improvements to the modern endoscope have been developed recently. The first one makes use of narrowband measurements (Narrow Band Imaging, NBI) consisting of selected ranges of the visible spectrum to increase the contrast [4]. Using multiple bands can increase the contrast of capillaries, and local application of dyes may be used for better imaging of cellular structures. Even though this technique is very recent, it is already being implemented in some clinical endoscopes.

Another recent advantage which will have a significant impact in endoscopy and its application in the clinic is the use of near-infrared fluorescence (NIRF) probes. These probes are still in pre-clinical development, where its potential has been demonstrated in animal models, particularly in murine models. It consist on the use fluorophores in the range of near infrared (Near Infrared Fluorescence, NIRF), coupled to specific antibodies or designed so that they are activatable, i.e. they remain in a quenched mode and emits fluorescence only in the presence of a specific molecular event, typically the presence of a specific enzyme $[5,6,7]$. These fluorescence measurements can be combined with both RGB videoscopic measures in real time and narrow band imaging (NBI) measurements, exponentially increasing the specificity of the technique.

Finally, an approach which may increase the low resolution exhibited by macroscopic measurements, is the use of confocal endomicroscopy. One of the latest developments in endoscopy has been the use of a bundle of thousands of fibers (between 10.000 and 150.000 in clinical applications) that are illuminated individually detecting the fluorescence emitted by each of these fibers sequentially, acting in effect as a confocal microscope. The small diameter of these fiber bundles allows its implementation through the working channel of a conventional endoscope (about $1 \mathrm{~mm}$ in diameter), adding microscopic cellular resolution measurements to the diagnostic endoscopy, in what is termed an "optical biopsy" [3]. Thus, the usefulness of the confocal endomicroscopy in early detection of tumors or structural alterations of the gastrointestinal mucosa has been demonstrated in several studies that were analyzed cases of Barrett's esophagus $[8,9]$, ulcerative colitis $[10,11]$ colorectal cancer $[12,13]$ or celiac disease [14].

However, the use of confocal endomicroscopy requires the use of contrast agents to provide a fluorescence signal, and one of its main disadvantages is the enormous amount of time which would require the study of large tissue areas. Currently, and pending on new contrast agent approvals for use in the clinic, there are some agents that can be administered in humans and provide fluorescence signal. These agents for clinical use allow observing tissue structures (Fluorescein), vascularization (Indocyanine Green) or even cell nuclei (Acriflavine).

Unfortunately, many of these developments today only apply in preclinical studies and some of them are in very early stages of development. The development of specific fluorescent markers emission spectrum in the near infrared (NIRF) has been a breakthrough in optical imaging in general and their possible application in biomedical research and clinical $[5,7]$. A particularly interesting type of fluorescent probes are molecules designed such that fluorescence is emitted only in the presence of a specific enzyme activity (as cathepsins or extracellular matrix metalloproteases). These agents, which have to be used in pre-clinical over 12 years research, are currently in the final stages of its assessment as agents for detection and diagnosis in humans. While the use of this type of fluorescent agents would represent a powerful new diagnostic tool, the fact is that few clinical current imaging techniques allow the detection of these markers.

\section{OBJECTIVES}

Anticipating the use of these markers in the clinic, a new generation of endoscopes that incorporate NIRF imaging capabilities is needed. The combination of a confocal endomicroscope with a fluorescence endoscope could provide the possibility of obtaining rapid and specific macroscopic information (through the use of fluorescent contrast agents), and a detailed analysis at the cellular level of the areas identified as potential injury.

Our objective is to design and implement and endoscope which is capable of taking video-rate images of both RGB and/or Narrow Band Imaging, combined with Near Infrared Fluorescence Imaging simultaneously. Additional to these macroscopic images, thorough the working channel of the endoscope we wan to introduce a fiber bundle coupled to the a 
scanning confocal unit, in order to provide confocal endomicroscopy information. Several problems will arise when implementing such a system, in particular: a) exposure times for the white light (RGB) images and the near infrared fluorescence images are different, but their data must be represented simultaneously; b) the confocal scanning unit must employ the least possible time to produce an image, in order to obtain crisp results and reduce the presence of unwanted movement; c) data must be stored in a fast and efficient manner, generating extremely large datasets in order to provide quantitative information. A schematic representation of our proposed design is shown in Figure 1, where they are depicted the macroscopic imaging unit with dual cameras and the confocal scanning unit entering through the working channel of an endoscope.

The main objective of our work is to develop this multi-scale system combining two modules compatible with conventional endoscopes; this assembly will allow the observation at cellular resolution of specific areas of interest through a fiber bundle and additionally macroscopic observation, and it be able to turn into advantage the potential NIRF fluorescent agents. The system will be developed and tested in animal models in the preclinical phase, identifying in the process which molecular markers provide greater contrast and significantly improve the sensitivity and specificity of diagnosis. In what follows we will detail the assembly of both modules and the design that controls the system, filters, pre-process the data and displays the images in real-time and offer the possibility of change the important parameters in order to obtain a wide range of operation modes. In addition a RGB/NIRF 3D image of the mouse intestine will be constructed.

\section{MATERIALS AND METHODS}

The multi-modal and multi-scale system is composed of two independent modules: a dual camera and dual excitation system for macroscopic imaging and a scanning endomicroscope, that work together in order to obtain both macroscopic and microscopic fluorescence images. Both modules are coupled to a conventional endoscope. Finally all these components are controlled through a custom software developed in Labview.

\subsection{Wide field module assembly}

The wide field NIRF Module (shown in figure 1) consist on a commercial endoscope (Hopkins II $0^{\circ} 1.9 \mathrm{~mm}$ Telescope, Karl Storz, Tuttlingen, Germany), which carries the image from the sample to the cameras. Two dichroic mirrors are used, one for combining the RGB and NIRF illumination and the other for splitting both images into the cameras (Edmund Optics, Barrington, NJ, USA). The images are projected onto the cameras through a lens (AC254-075, Thorlabs Inc, Delaware, USA) maximizing the coverage of the CMOS sensor (Thorlabs, Newton, NJ, USA) so that one camera is capturing the visible field and the other camera the fluorescence emitted by the contrast agent. The cameras (Manta G-145 Color and Manta G-145 NIR, Allied Vision technologies, Stadtroda, Germany) are working at $30 \mathrm{fps}$ video rate so it is a real-time acquisition. The illumination consist on a broadband halogen lamp (OSL2, Thorlabs, Newton, NJ, USA) properly coupled to the endoscope and a laser for the excitation of the fluorophores. It is worth mentioning that a set-up without the laser illumination could be assembled by placing the appropriate excitation shortpass filter on the halogen illuminator, so it will be illuminating the sample with white light and exciting the fluorophores through a specific excitation filter. The endoscope is carrying both illumination from the lamp to the sample and the images from the sample to the cameras simultaneously. All the optical components were assembled and mounted on portable optical breadboards using conventional optomechanics (Thorlabs, Delaware, USA). 


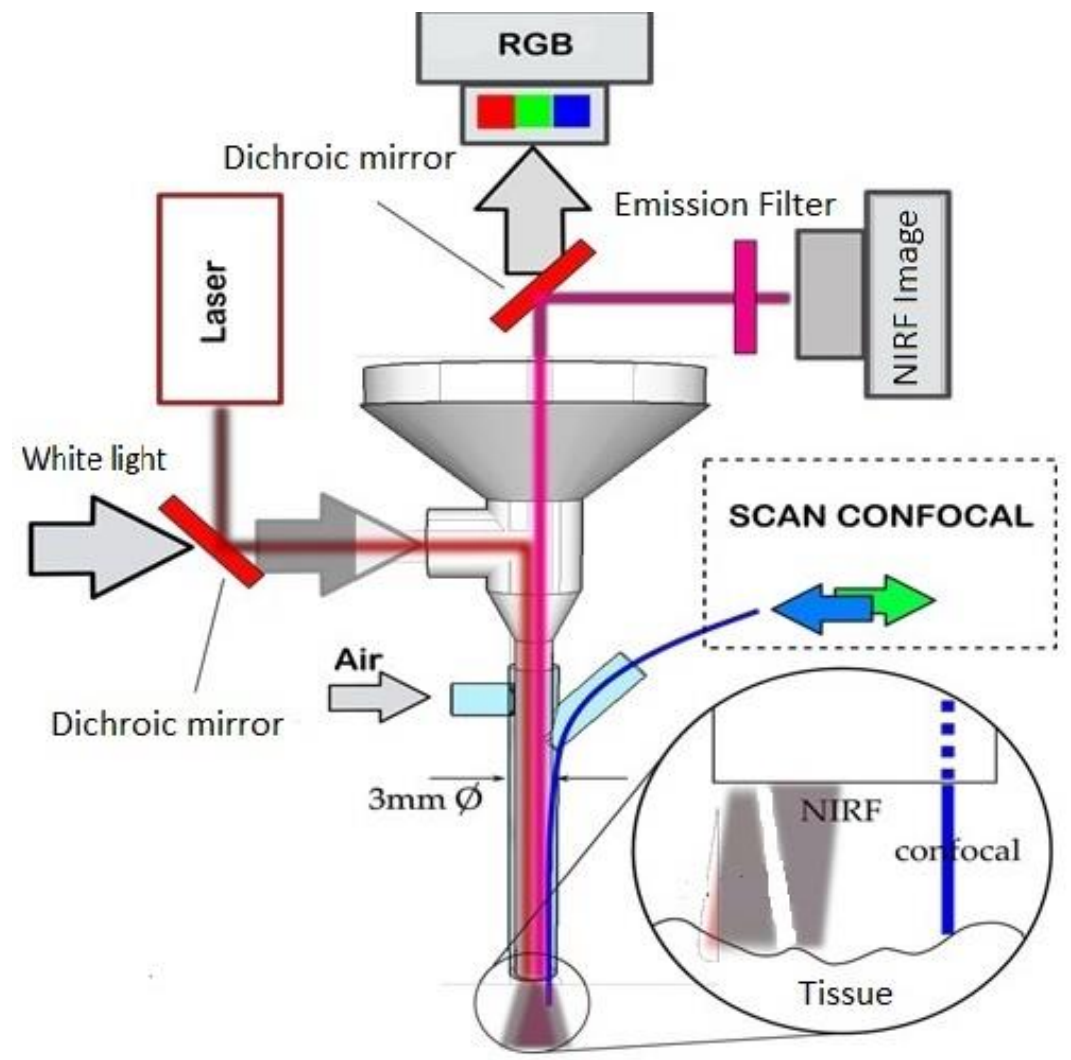

Figure 1: Scheme of the wide field NIRF/Confocal endomicroscopy system

\subsection{Confocal endomicroscopy module assembly}

The Confocal module (Figure 2) is based on the principles of confocal microscopy, but with the particularity that now the main element is a fiber bundle (FIGH-30-850N, Fujikura, Kōtō, TKY, Japan). A laser diode module (CPS520, Thorlabs Inc, Delaware, USA) is used to illuminate the sample, making use of 2D galvanometer mirrors (GVS012, Thorlabs Inc, Delaware, USA) to scan the fiber bundle (30.000 fibers), which carries the illumination to the sample and collects the fluorescence back to the photodiode (APD130A2, Thorlabs Inc, Delaware, USA). After the mirrors, a scan lens (LSM02, Thorlabs Inc, Delaware, USA), a relay lens (AC254-030, Thorlabs Inc, Delaware, USA) and an objective (Plan Apochromat 10X, Motic, Weztlar, Germany) are used. This lens system will focus the laser on each individual fiber so that the galvo scanning illuminates each fiber independently. A dichroic mirror and an emission filter (Edmund optics, Newport, NJ, USA) are also used in order to receive only fluorescence light on the photodiode. The fluorescence light is focused onto a pinhole (P25S, Thorlabs Inc, Delaware, USA) which will block all the light that is not coming from the illuminated fiber, avoiding fluorescence signal coming from other points of the tissue and increasing the system resolution. The light exiting the pinhole is finally collected by the avalanche photodiode. All the optical components were assembled using a $30 \mathrm{~mm}$ cage assembly optomechanics (Thorlabs, Delaware, USA). The resolution of the system is limited by the core-to-core distance of the fiber bundle, which in this case is $4.5 \mu \mathrm{m}$, while the field of view is limited to the bundle diameter $(1 \mathrm{~mm})$.

The endoscope examination sheath (STZ 61029D, Karl Storz, Tuttlingen, Germany) admits the Hopkins optic endoscope and also has a work channel of $1 \mathrm{~mm} \varnothing$ for the fiber bundle, so both macroscopic and microscopic images can be acquired at the same time and the position of the fiber bundle can be monitored from the wide field image. 


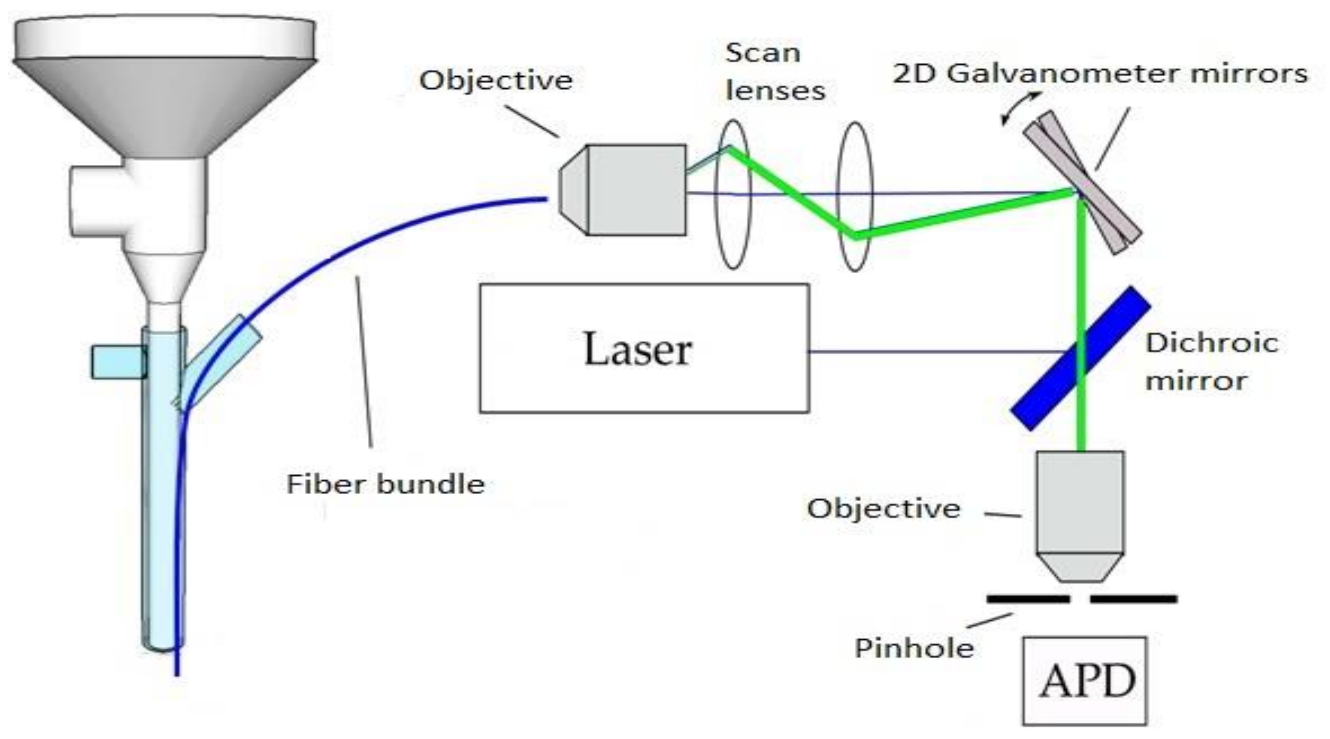

Figure 2: Scheme of the confocal scanning endomocroscopy module

\subsection{Software}

The software implemented for both modules of the system has been developed with Labview (National Instruments, Austin, TX, USA), and includes a user-friendly, performing all the tasks related with hardware control and data acquisition (see Figure 3), processing and displaying images, and enabling the user to record and save them automatically. All the relevant parameters can be changed for different acquisition modes (fast-scan or point by point raster scan, step size, etc).

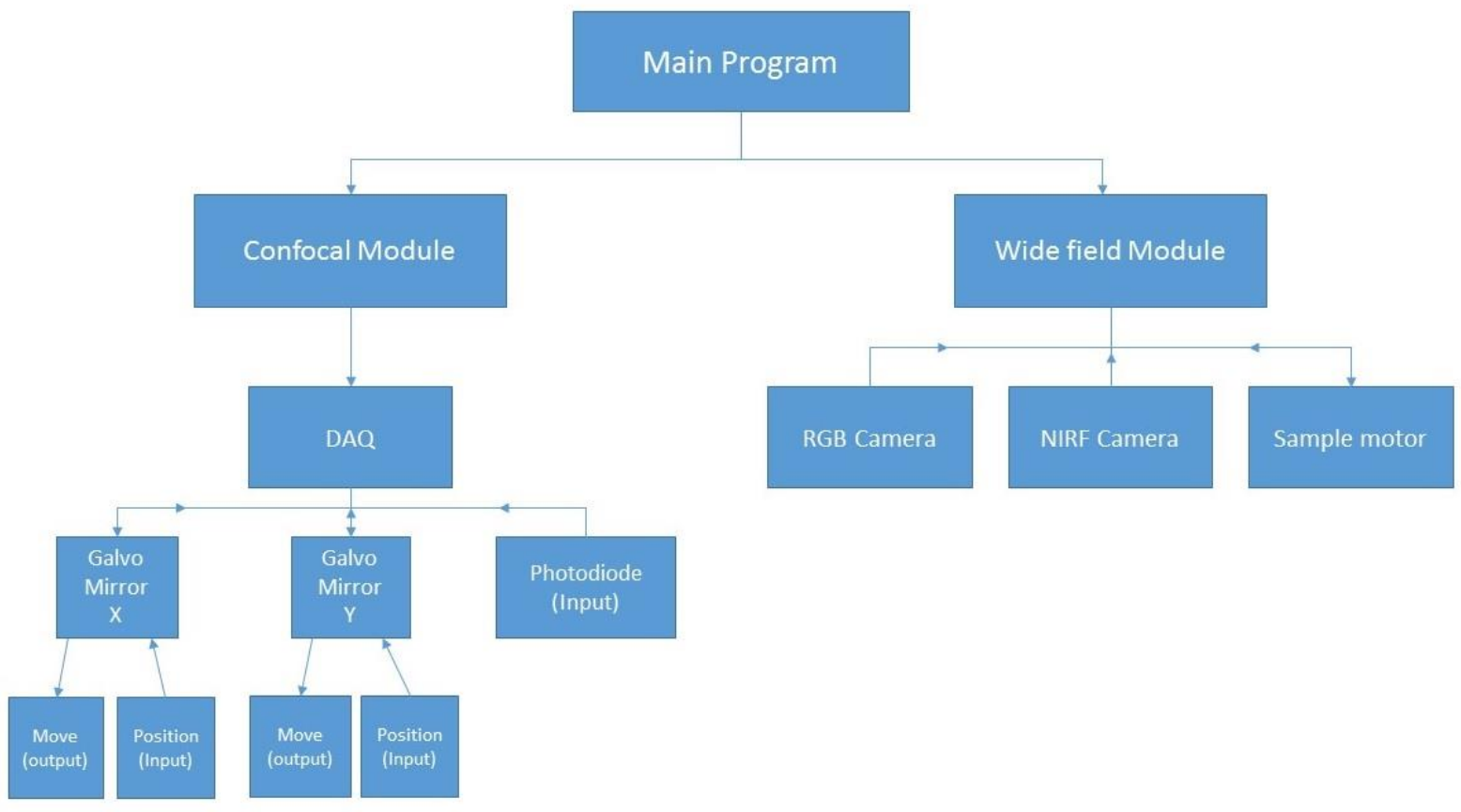

Figure 3: Diagram of the software components 
The first part of the software applies to the wide field endoscope. It is simpler than the Confocal unit control, consisting on the acquisition of both NIRF and RGB images delivered by the cameras, its filtering and processing for its final combination so that the visible and near infrared fluorescence images are displayed overlayed on the same image. In addition, this module controls the motor for specimen positioning and the collection of multiple images at a given rate for data post-processing.

The second part of the software is dedicated to the confocal module. Here the most challenging part is the scanning method of the mirrors and its synchronization with the photodiode measurements, since this will determine the important parameters of the system as time rate of acquisition, resolution and field of view. The Data Acquisition Card (DAQ) (NI 6009, National Instruments, Austin, TX, USA) which communicates with the three devices and the PC lag time determine the time required to obtain a full scan.

The software permits to acquire images in different conditions in order to operate in coordination with the sample requirements and three scanning methods: manual, fast and slow (this last two will be analyzed on the results section).

\subsection{Sample positioning}

In order to obtain accurate information on the location of the endoscope, the specimen is mounted onto a micrometer precision motor (TL-S Series Motorized Linear Stage, Zaber Technologies Inc, Vancouver, Canada) (as shown in figure 4) in order to control the penetration of the endoscope and observe different regions of the intestine so a detailed exam of each region can be done. Note that for each of these regions images of both NIRF and RGB data are obtained and stored for post-processing.

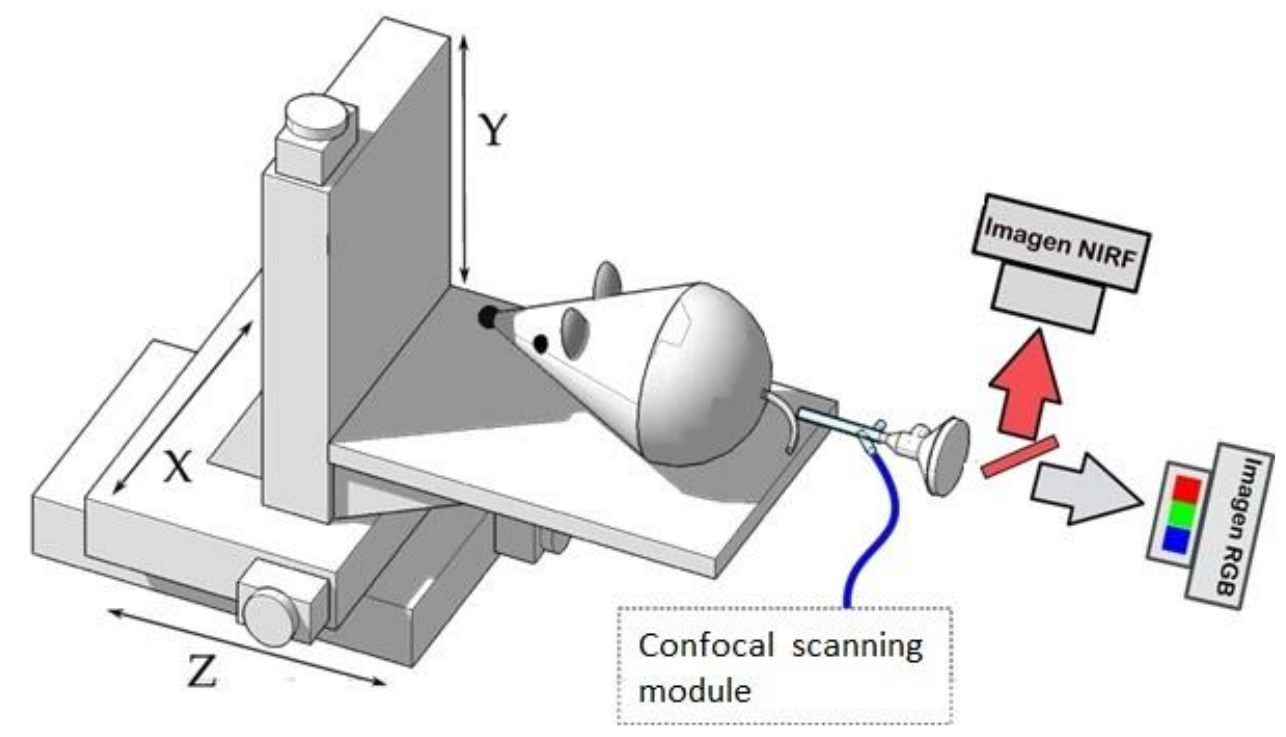

Figure 4: Experimental set up for endoscopy in mouse and rat models.

\section{RESULTS}

A 30fps video rate wide field image which combines RGB and NIRF is displayed by the software (Figure 5), allowing the detection and mapping of the desired regions of tissue. This is a fast and superficial exam that will inform about possible lesions for the subsequent detailed examination with the microscopic module for higher resolution -local imaging of the region of interest. 


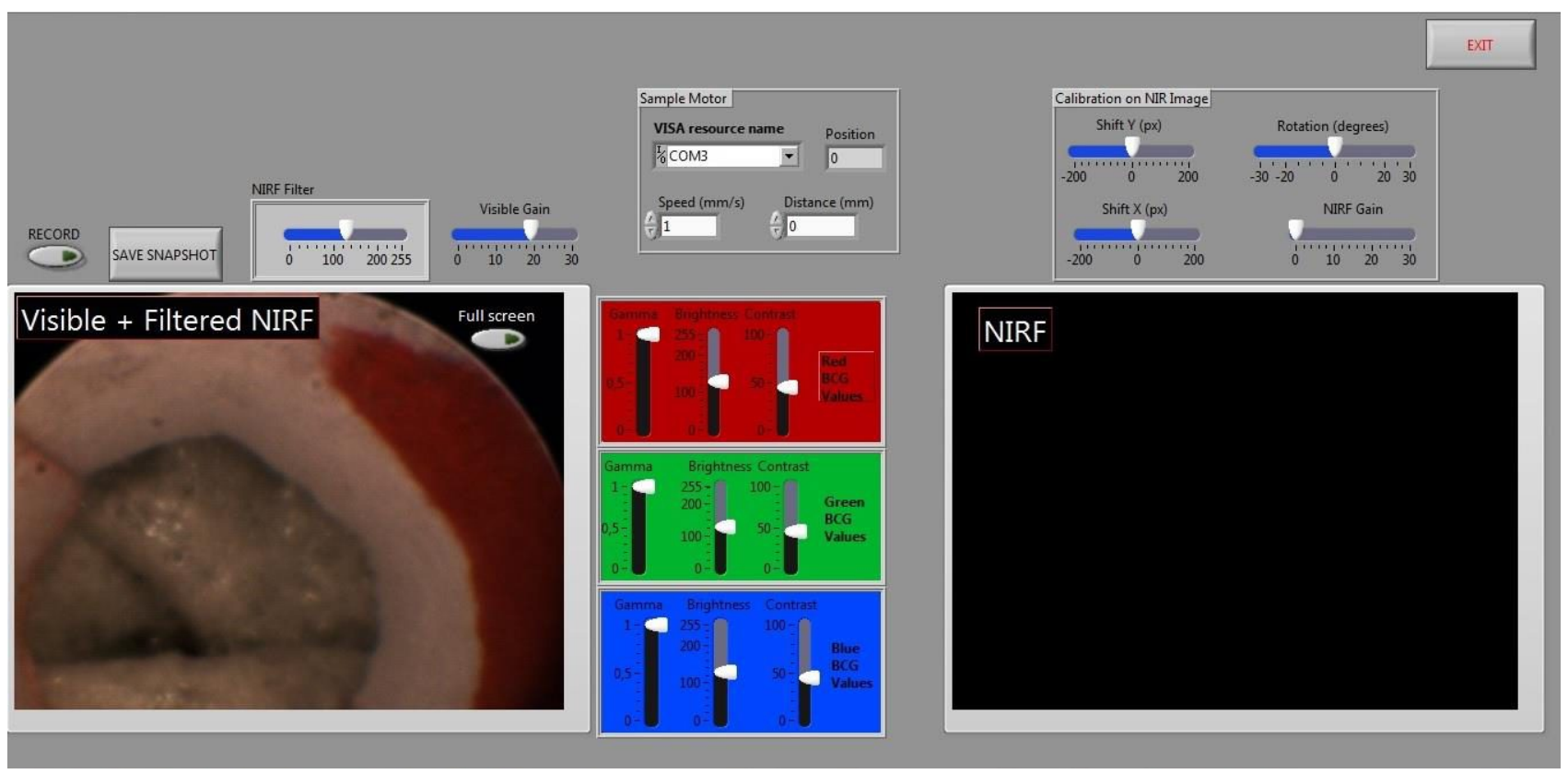

Figure 5: Interface of the wide field endoscope module showing image of a phantom not exhibiting fluorescence in the Near Infrared.

Two scanning methods have been studied in order to obtain a high-resolution image and a fast rate of acquisition (Figure 6). As in all imaging scenarios, there is a compromise between the field of view, the resolution and the acquisition time. However, since our endoscope includes the macroscopic module that offers a wide field image, the scanning FOV can be reduced and the selection of the area measured is done by placing the fiber in contact with the tissue while monitoring this from the cameras. With respect to the FOV of our fiber, the FOV is given by the fiber bundle diameter (1mm in our case), so several hundreds of cells can be imaged.

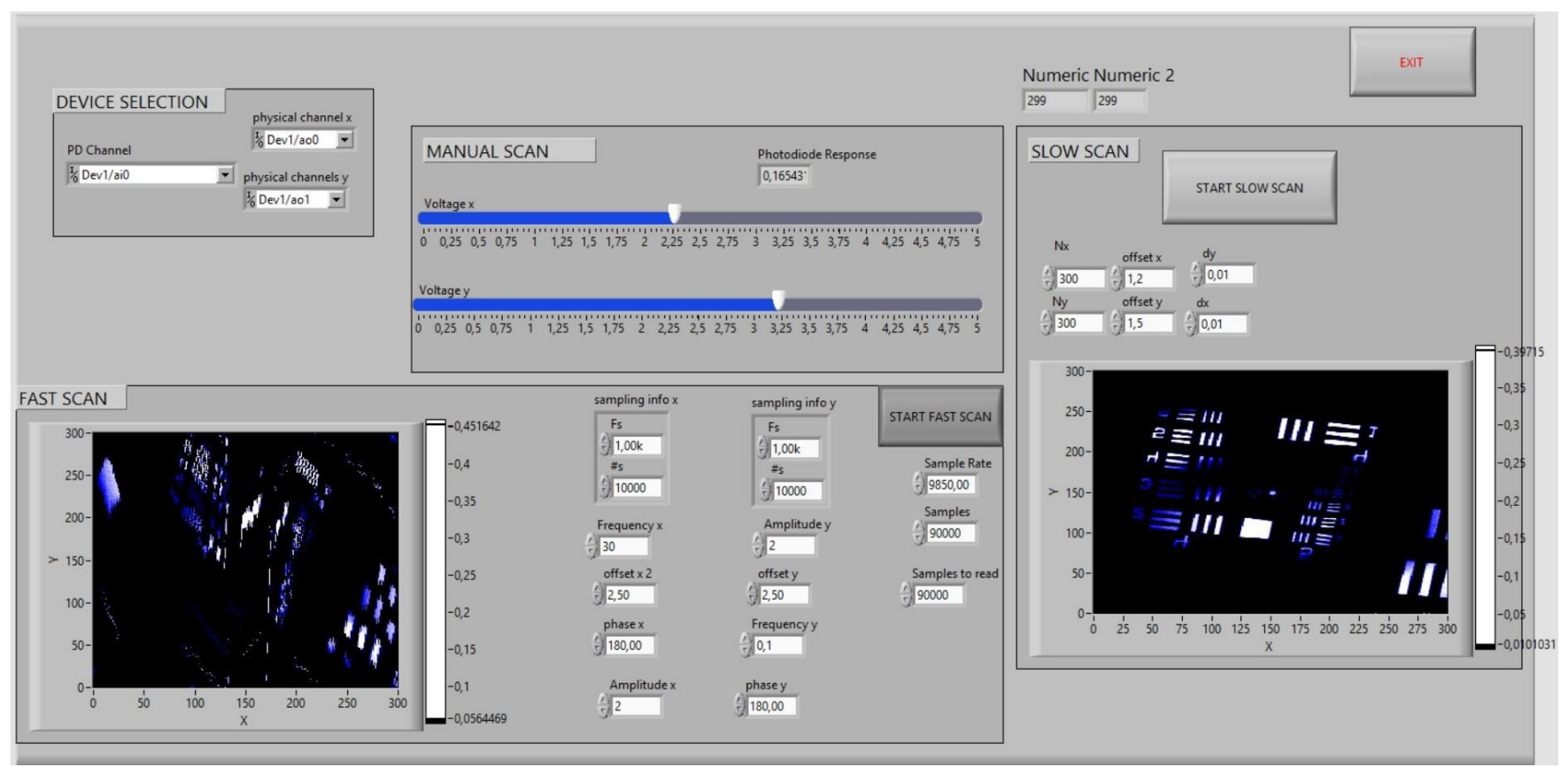

Figure 6: Interface of the confocal module software that shows the distortion caused on the fast scan (left) and a image of an USAF 1951 target acquired with the slow scan. 
The first method is a slow scan, which consists on moving the galvanometer mirrors to a certain position and then taking a measurement with the photodiode. This process is time consuming and not practical for generating a high-resolution image, but the error in the position of the mirrors and the synchronization with the photodiode is minimum (see Table 1).

The second method is a fast scan, which moves the mirrors scanning a squared area of $1 \mathrm{~mm}^{2}$ independently of the photodiode measurements. In this case the position of the galvanometer mirrors needs to be known accurately when the photodiode triggers is needed. Once the intensity values and their corresponding coordinates are known and stored, the image can be constructed by applying a scattered data interpolation based on Delaunay triangulation It is worth mentioning that the acquisition rate is an extremely important parameter for in-vivo cellular resolution imaging, due to displacement due to breathing and inherent cell motility.

Table 1: comparison between fast and slow scanning and its characteristic parameters

\begin{tabular}{|c|c|c|c|c|}
\hline Scanning type & FOV & Resolution & Points & Time \\
\hline \multirow{3}{*}{ Fast Scan } & $1 \mathrm{~mm}^{2}$ & $3 \mu \mathrm{m}$ & $400 \times 400$ & $6 \mathrm{~s}$ \\
\cline { 3 - 5 } & & $4 \mu \mathrm{m}$ & $300 \times 300$ & $5 \mathrm{~s}$ \\
\cline { 2 - 5 } & $1 \mathrm{~mm}^{2}$ & $8 \mu \mathrm{m}$ & $150 \times 150$ & $3 \mathrm{~s}$ \\
\cline { 3 - 5 } & & $12 \mu \mathrm{m}$ & $100 \times 100$ & $2 \mathrm{~s}$ \\
\hline \multirow{3}{*}{ Slow Scan } & $1 \mathrm{~mm}^{2}$ & $3 \mu \mathrm{m}$ & $400 \times 400$ & $100 \mathrm{~s}$ \\
\cline { 3 - 5 } & & $4 \mu \mathrm{m}$ & $300 \times 300$ & $60 \mathrm{~s}$ \\
\cline { 2 - 5 } & $1 \mathrm{~mm}^{2}$ & $8 \mu \mathrm{m}$ & $150 \times 150$ & $15 \mathrm{~s}$ \\
\cline { 3 - 5 } & & $12 \mu \mathrm{m}$ & $100 \times 100$ & $10 \mathrm{~s}$ \\
\hline
\end{tabular}

\section{CONCLUSION AND PERSPECTIVES}

A multi-scale high-resolution endoscopy system and its software have been developed, and its speed characterized in order to optimize the results for in-vivo imaging. Our system is currently capable of combining Near Infrared Images and RGB images, together with high resolution endomicroscopy images. The next step is to apply this setup to in-vivo imaging of animal models, in particular gastro intestinal intestine diseases in murine models will be carried out at both macroscopic and microscopic scales, using the endoscope for both macro- and microscopic imaging.

Further improvements of the imaging system in order to improve the resolution of the microscopy images include the use of a more powerful laser (for collimation and beam shaping) with a lower $\mathrm{M}^{2}$ factor. To reduce the acquisition time a higher speed DAQ card could be used. Additionally, new scanning procedures are under evaluation.

\section{ACKNOWLEDGMENTS}

The authors acknowledge support from EC FP7 IMI project PREDICT-TB, the EC FP7 CIG grant HIGHTHROUGHPUT TOMO, the Spanish MINECO project grant FIS2013-41802-R MESO-IMAGING, and TOPUS S2013/MIT-3024 project from the regional government of Madrid.

\section{REFERENCES}

[1] Benjamin A Flusberg, Eric D Cocker, Wibool Piyawattanametha, Juergen C Jung, Eunice L M Cheung \& Mark J Schnitzer, "Fiber-optic fluorescence imaging," Nature Publishing Group, (2005). 
[2] Gyungseok Oha, Euiheon Chung Seok H. Yun, "Optical fibers for high-resolution in vivo microendoscopic fluorescence imaging,” Optical Fiber Technology, Volume 19 Issue 6 Part B 760-771 (2013).

[3] Mark Pierce, Dihua Y, Rebecca Richards-Kortum, "High-resolution Fiber-optic Microendoscopy for in situ Cellular Imaging," Journal of Visualized Experiments, (2011).

[4] Nadhi Thekkek, Sharmila Anandasabapathy and Rebecca Richards-Kortum, "Optical molecular imaging for detection of Barrett's-associated neoplasia,” World J Gastroenterol, 17(1): 53-62, (2011).

[5] Ralph Weissleder, Brian D. Ross, Alnawaz Rehemtulla, Sanjis S. Gambhir, [Molecular Imaging: principles and practice], PMPH-USA, (2010).

[6] Peiman Habibollahi, Jose-Luiz Figueiredo, Pedram Heidari, Austin M Dulak, Yu Imamura, Adam J. Bass, Shuji Ogino, Andrew T Chan, Umar Mahmood, "Optical Imaging with a Cathepsin B Activated Probe for the Enhanced Detection of Esophageal Adenocarcinoma by Dual Channel Fluorescent Upper GI Endoscopy," Theranostics, 2(2):227234, (2012) doi:10.7150/thno.4088.

[7] Martin A. Funovics, Herlen Alencar, Henry S. Su, Khashayarsha Khazaie, Ralph Weissleder, and Umar Mahmood, "Miniaturized Multichannel Near Infrared Endoscope for Mouse Imaging," Molecular Imaging, Vol. 2 No. 4 350-357 (2003).

[8] Kiesslich R, Gossner L, Goetz M, Dahlmann A, Vieth M, Stolte M, Hoffman A, Jung M, Nafe B, Galle PR, Neurath MF, "In vivo histology of Barrett's esophagus and associated neoplasia by confocal laser endomicroscopy," Clin Gastroenterol Hepatol, 4: 979-987 (2006).

[9] Dunbar KB, Okolo P, Montgomery E, Canto MI, "Confocal laser endomicroscopy in Barrett's esophagus and endoscopically inapparent Barrett's neoplasia: a prospective, randomized, double-blind, controlled, crossover trial," Gastrointest Endosc 70: 645-654 (2009).

[10] Kiesslich R, Goetz M, Lammersdorf K, Schneider C, Burg J,Stolte M, Vieth M, Nafe B, Galle PR, Neurath MF, "Chromoscopy-guided endomicroscopy increases the diagnostic yield of intraepithelial neoplasia in ulcerative colitis," Gastroenterology, 132: 874-882 (2007).

[11] Hurlstone DP, Thomson M, Brown S, Tiffin N, Cross SS, Hunter MD, "Confocal endomicroscopy in ulcerative colitis: differentiating dysplasia-associated lesional mass and adenoma- like mass," Clin Gastroenterol Hepatol, 5: 12351241 (2007).

[12] Polglase AL, McLaren WJ, Skinner SA, Kiesslich R, Neurath MF, Delaney PM, “A fluorescence confocal endomicroscope for in vivo microscopy of the upper- and the lower-GI tract," Gastrointest Endosc, 62: 686-695 (2005).

[13] Kiesslich R, Burg J, Vieth M, Gnaendiger J, Enders M, Delaney P, Polglase A, McLaren W, Janell D, Thomas S, Nafe B, Galle PR, Neurath MF, "Confocal laser endoscopy for diagnosing intraepithelial neoplasias and colorectal cancer in vivo," Gastroenterology, 127: 706-713 (2004).

[14] Venkatesh K, Abou-Taleb A, Cohen M, Evans C, Thomas S, Oliver P, Taylor C, Thomson M, "Role of confocal endomicroscopy in the diagnosis of celiac disease.," J Pediatr Gastroenterol Nutr, 51: 274-279 (2010).

[15] Joshua A. Udovich, Andy R. Rouse, Anthony Tanbakuchi, Molly A. Brewer, Richard Sampliner, Arthur F. Gmitro, "Confocal microendoscope for use in a clinical setting," Proc. of SPIE, Vol. $643264320 \mathrm{H}$ (2007) doi:

10.1117/12.701798.

[16] Matthew D. Risi, Houssine Makhlouf, Andrew R. Rouse, Anthony A. Tanbakuchi and Arthur F. Gmitro, "Design and Performance of a Multi-Point Scan Confocal Microendoscope," Photonics, 1 421-431 (2014),

doi:10.3390/photonics1040421. 
[17] Cameron M. Lee, Christoph J. Engelbrecht, Timothy D. Soper, Fritjof Helmchen and Eric J. Seibel, "Scanning fiber endoscopy with highly flexible, $1 \mathrm{~mm}$ catheterscopes for wide-field, full-color imaging, " J. Biophoton, 3 No. 5-6 385407 (2010), DOI 10.1002/jbio.200900087.

[18] Wibool Piyawattanametha, Hyejun Ra, Zhen Qiu, Shai Friedland, Jonathan T. C. Liu, Kevin Loewke, Gordon S. Kino, Olav Solgaard, Thomas D. Wang, Michael J. Mandella and Christopher H. Contaga, "In vivo near-infrared dualaxis confocal microendoscopy in the human lower gastrointestinal tract," Journal of Biomedical Optics, 17(2) 021102 (2012).

[19] Zhen Qiu, Zhongyao Liu, Xiyu Duan, Supang Khondee, Bishnu Joshi, Michael J. Mandella, Kenn Oldham Katsuo Kurabayashi and Thomas D. Wang, "Targeted vertical cross-sectional imaging with handheld near-infrared dual axes confocal fluorescence endomicroscope,” Biomedical Optics Express, Vol. 4 No. 2322 (2013). 